\title{
Fluorometholone Acetate Ophthalmic Suspension
}

National Cancer Institute

\section{Source}

National Cancer Institute. Fluorometholone Acetate Ophthalmic Suspension. NCI

Thesaurus. Code C116751.

An ophthalmic suspension containing the acetate form of fluorometholone, with potential anti-infective and immunosuppressive activities. Upon ocular administration, fluorometholone binds to glucocorticoid receptors in the cytoplasm and forms a receptor-lig and complex, which translocates to the nucleus where it binds to glucocorticoid response elements (GRE) in the promoter region of target genes. This leads to the expression of specific target genes that control inflammation. Specifically, by inducing the expression of lipocortins that inhibit the enzymatic activity of phospholipase A2, the release of arachidonic acid is blocked, which prevents the production of inflammatory mediators, and inflammatory-induced ocular swelling, redness and itching. 